Review Article

Open Access

\title{
Cooking for Individuals with Food Allergies and Intolerances
}

Tiffany LaLonde ${ }^{1}$ and Ravi Jadeja ${ }^{2 \star}$

IRobert M. Kerr Food and Ag Products Center, Oklahoma State University, Stillwater, Oklahoma, USA

2 Department of Animal Science, Oklahoma State University, Stillwater, Oklahoma, USA

\section{Article Info}

\author{
*Corresponding author: \\ Ravi Jadeja \\ Assistant Professor and \& Food Safety \\ Specialist \\ 106 FAPC \\ Robert M. Kerr Food \& Ag Products Center \\ Oklahoma State University \\ Stillwater, OK, 74078 \\ USA \\ Tel: 405-744-3922 \\ E-mail: Ravi.Jadeja@okstate.edu
}

\section{Received: May 2, 2018 \\ Accepted: May 14, 2018 \\ Published: May 19, 2018}

Citation: LaLonde T, Jadeja R. Cooking for Individuals with Food Allergies and Intolerances. Madridge J Food Technol. 2018; 3(1): 114-120.

doi: $10.18689 / \mathrm{mjft}-1000117$

Copyright: (c) 2018 The Author(s). This work is licensed under a Creative Commons Attribution 4.0 International License, which permits unrestricted use, distribution, and reproduction in any medium, provided the original work is properly cited.

Published by Madridge Publishers

\begin{abstract}
Due to the economic downturn in 2008 and the public's growing demand to purchase locally produced foods, a strong grass-roots movement to pass state cottage food laws has allowed opportunities for small-scale food businesses to become established out of homes. Purchasing food items from a local home producer implies a perceived feeling of safe and wholesome food because it was prepared in someone's home. However, this not always the case and the safety of the home cooked food depend on the producer's food safety knowledge. Unfortunately, there are not many affordable food safety training and guides available for home food producers. This review is intended to serve as a comprehensive guide for very small producers to prepare food for individuals with food allergies and intolerances.
\end{abstract}

Keywords: Allergies; Food Intolerances; Egg; Dairy; Flour.

\section{Introduction}

Food allergies and intolerances are a fast growing health concern in today's growing populations. A food allergy is defined as an adverse health effect resulting from a specific immune response that occurs reproducibly on exposure to a given food [1]. A food intolerance (also known as a food sensitivity) is defined by the American Academy of Allergy Asthma and Immunology as, "[occurring] when a person has difficulty digesting a particular food" [2]. The major difference is allergies involve an immune system response, whereas an intolerance/sensitivity involves the digestive system.

The CDC reports that between the years 1997-1999 and 2009-2011 food allergy prevalence increased by 50 percent [3]. According to a study on self-reported allergies, the instances of childhood allergies has increased as follows among certain ethnic groups per decade: 2.1 percent among African Americans, 1.2 percent among Hispanics, and 1 percent among whites. Approximately 15 million people in the United States suffer from food allergies; more than 9 million adults (roughly 4 percent or people aged 18 or older), and 5.9 million children [4-6]. Among children, this is equivalent to 1 in 13, or two in every classroom $[4,7]$. Of children suffering from food allergies, 30 percent have more than one food to which they are allergic $[4,8,9]$.

Food allergies can cause a major impact on day to day lives and activities of not only those who suffer from food allergies but their friends and families. Many parents who have recently diagnosed children choose to restrict social activities, travel and eating in restaurants [10]. Parents surveyed during food allergy appointments reported the following: more than one quarter - their children do not participate in sleepovers or camps, more than fifteen percent do not eat in restaurants, more than ten percent avoid childcare or playdates at friends' homes, and ten percent choose to home-school their children [11]. 
While food allergies are more common among children, an allergy can develop at any time, at least fifteen percent of food allergies are first diagnosed among adults [4]. Teenagers and young adults with food allergies are among the highest risk for fatal food-induced anaphylaxis $[8,9,12]$. More than 170 foods have been reported to cause reactions in the United States [1]. The United States recognizes eight major allergens - Milk, Egg, Peanut, Tree Nut, Wheat, Soy, Fish, and Crustacean (Shellfish) [1,13]. The following will address substituting ingredients for some of these allergens. When substituting items in recipes, it is always trial and error. While this list is a guide, it is not an extensive or exhaustive resource. Some of these substitutes may not work depending on individual tolerances to the ingredients, as well as the composition of the item being made.

\section{Egg}

Egg substitution is fairly straight forward, the majority of substitutes are 1:1. However, it is important to note that not every ingredient is appropriate for every item. When a recipe calls for two or more eggs, it can sometimes be beneficial to combine two different egg substitutes. Since eggs offer a variety of different qualities when it comes to recipes, it is important to identify how the egg will be acting within the recipe and substitute accordingly [14] (Figure 1).

Moistening: Fruit/Veggie Purees, Tofu

Leavening: Ener-G egg replacer (may need to add additional baking powder), Fruit/veggie

purees, Non-Dairy Mayo/Yogurt, Tofu

Binder: Flax or Gelatin "eggs", Chia seeds, Banana/Sweet Potato

\section{Richness: Flaxmeal, Chia Seeds, Fruit Puree}

Here are a few other rules of thumb when it comes to substituting for eggs:

- For a crunchier cookie use a starch (Ener-G Egg Replacer)

- For a softer/chewier cookie, use a fruit puree (applesauce, pumpkin, sweet potato, etc.)

- Flaxmeal or Chia seeds can impart a "nutty" flavor

- Tofu, Non-Dairy Mayo, Non- Dairy Yogurt are high in fat

\section{Basic overview of Egg Substitutes:}

- Cookies, Brownies, Bars: Ener-G Egg Replacer, Flaxseed Meal, Tofu, Gelatin "egg."

- Cakes, Muffins, Quick Breads: Ener-G Egg Replacer, Chia Seeds, Vinegar+Baking Soda+Non-Dairy Milk, Fruit/ Veggie Puree, Non-Dairy Mayo or Yogurt, Applesauce, Tofu, Gelatin "egg."

- Yeast Breads: Chia Seeds, Psyllium, Veggie Puree, Ener-G Egg Replacer [15].

\section{Dairy}

Substituting for dairy is only more challenging because there are more products within the dairy category. However, there are a large variety of options available.
Figure 1. How to substitute egg with commonly available ingredients.

1 Egg:

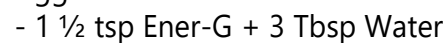

- 1 Tbsp flax meal + 3 Tbsp Water

- 1 Tbsp Ground Chia + 3 Tbsp Water

- 1 Tbsp Psyllium + 3 Tbsp Water

$-1 / 2$ Tbsp Vinegar/ACV + $1 / 2$ tsp Baking Soda (may need additional baking powder)

- $1 / 4$ C Mashed Banana Puaree

$-1 / 4-1 / 3$ C Applesauce (+1 tsp Baking Powder)*

$-1 / 4-1 / 3 C$ Any Puree (Pumpkin, Sweet potato, prune, pear, etc.)*

- $1 / 4$ C Tofu (blended)

- $1 / 4$ C Non-dairy Yogurt

- $1 / 4$ C Non-dairy Mayo

- 1 Tbsp Gelatin +3 Tbsp Water

- 2 Tbsp Arrowroot starch (cakes only)

- $1 / 4$ C Vegetable Oil (cakes/cookies only)

* Depending on the recipe sources use different amounts, or add baking powder

\section{Milk}

When substituting for milk in baked goods, it is important to take into account the fat content of the milk being used to substitute. The fat allows for better texture and flavor, but can also assist with rising in baked good products. There are a variety of substitutes for cow's milk: goat's milk, soy milk, rice milk, almond milk, oat milk, and coconut milk are fairly common and can be both store-bought or home-made. This is not an exhaustive list, but merely the most commonly seen in stores. They can all typically be subbed on a 1:1 basis. Soy milk is thicker, has a more pronounced flavor and works well in baked goods. Rice milk is more delicate, and many say comes closest to a cow's milk in flavor and could be used as a suitable substitute for dairy milk in many recepies. Oat milk has a light flavor and very milk sweetness; it substitutes best for low-fat, or fat-free milk. Coconut milk is a good substitute in many recipes because of its high-fat content. However, it is known to impart flavor onto whatever it is being used in, so that is important to keep in mind $[16,17]$.

Buttermilk: A simple mixture of 1 cup dairy free milk +2 Tbsp of vinegar or lemon juice will create a great substitute for buttermilk, if left to sit for 5 minutes.

Evaporated Milk: Evaporated milk is typically just milk that has its water content reduced by $60 \%$. So by taking 3 cups of a dairy-free milk and cooking over medium-low heat (taking care not to scald), while stirring occasionally, will create a dairy-free evaporated milk. Coconut milk can also work in place of evaporated milk, with a slight flavor impact.

Sweetened Condensed Milk: This is simply evaporated milk that has been sweetened with enough sugar to create more of a syrup. To create a dairy free version simply take your evaporated milk and add $1 \frac{1 / 4}{4}$ Cup maple syrup or 1 Cup of honey or granulated sugar [18].

Butter/Margarine: There are a variety of options when substituting for butter/margarine, depending on personal preferences and recipes. Some standard options include: palm oil/shortening, lard, Non-Dairy Butter (ex: Earth Balance or Fleischmanns), oils (vegetable, canola, coconut, etc.), 
mashed banana, avocado and applesauce. All but olive oil, are a 1:1 swap. Olive oil requires slightly less per amount of butter/margarine (Figure 2).

Figure 2. How much olive oil should be used to replace butter/ margarine in the recipes?

\begin{tabular}{|ll|}
\hline Butter/Margarine & Olive Oil \\
1 tsp & $3 / 4$ tsp \\
1 Tbsp & $21 / 4$ tsp \\
2 Tbsp & $41 / 2$ tsp \\
$1 / 4$ Cup & 3 Tbsp \\
$1 / 3$ Cup & $1 / 4$ Cup \\
$1 / 2$ Cup & $1 / 4$ Cup +2 Tbsp \\
$2 / 3$ Cup & $1 / 2$ Cup \\
$3 / 4$ Cup & $1 / 2$ Cup +1 Tbsp \\
1 Cup & $3 / 4$ Cup \\
\hline
\end{tabular}

It is important to note that many non-dairy butters have a higher water content, so in a heavy butter recipe, the results may be a little different. When using coconut oil, a refined version will impart no coconut flavor. Also, olive oils have varied degrees of flavor that can transfer into/onto dishes [19].

It is important to note that many non-dairy butters have a higher water content, so in a

Creams: Replacing creams in recipes can be a little trickier, it is necessary to decide on a flavor profile first and work from there. For savory items, cashew "cream", white bean puree, avocado, cauliflower/potato/squash puree are great options. For sweeter dishes, coconut milk/cream and coconut/almond/ soy based creamers are good options. Also mixing $2 / 3$ Cup dairy-free milk with $1 / 3$ Cup melted dairy free margarine can be substituted.

Whipping Cream: To create a dairy-free whipping cream, you can either use a dairy-free store bought brand (Ex: So Delicious, Rice Whip, Soy Whip, etc.). Or you can make your own using coconut cream.

Sour Cream: To replace sour cream you can use any dairyfree yogurt or make your own using coconut milk and probiotic powder.

\section{Flour}

Tackling gluten-free flours can be an overwhelming task. Trying to find the perfect combination of flours to sub for wheat flour can be a challenge. These flours can be expensive, and so it can be frustrating to use the flours in your recipes only to have them not turn out as expected. Once the properties of each of the flours are understood, then creating a mixture of flours to substitute in recipes is a little easier $[20,21]$.

Bean/Legume Flours: Beans and legumes are natural sources of protein, fiber, calcium and folate. They are packed with antioxidants and are very nutrient dense.

Chickpea (Garbanzo)/Bean (Navy/Pinto/Red)/Soy: These flours work well with bread, pizzas and spice cakes. They can cause an aftertaste so it may be necessary to offset this flavor by only using up to 30 percent in blends and use in recipes that contain other powerful flavors such as brown sugar, molasses, chocolate, etc. Bean flours are not suitable for delicately flavored items. Adding a small amount $(1 / 4-1 / 2 C)$ to pie crusts or wraps can add some elasticity. These flours are also very good at assisting with rising in baked goods. They can also be used as thickening agents [22,23].

STORAGE: Room temperature or Refrigeration.

USAGE: Up to 30 percent of total flour blend.

Pea/Green Pea: These are very similar to bean flours, but lack the aftertaste. Pea flours lend structure to baked goods. Green pea flour can create a green hue onto final products, which may not be suitable for items that are typically white. They can also give a starchy taste if too much is used in a blend.

STORAGE: Room temperature or Refrigeration

USAGE: Up to 30 percent of total flour blend

Grains: Some grain flours can cause cross-reactivity in individuals with celiac disease or gluten intolerances [24].

Amaranth: This dense flour is high in protein, calcium, and iron. They can add structure and aid in the browning of baked goods. If too much amaranth is used it can create a bitter aftertaste, so use sparingly. It works great as a thickener for sauces or pie fillings that have a strong flavor to overcome the flavor of the amaranth $[25,26]$.

STORAGE: Refrigerate in an airtight container.

USAGE: $10-20$ percent of flour blend, but no more than $1 / 2$ Cup per recipe

Corn: Flours made from corn vary in degree of coarseness, with corn meal being the coarsest, followed by masa harina, and the finest being corn flour. (US made corn flour is different than "corn flour" in Great Britain. The British "corn flour" is actually what Americans call corn starch). Any of the coarser grinds can be ground finer in a food processor. Corn flours come in both white and yellow varieties and have a slightly nutty taste. They are good sources of fiber, riboflavin, niacin, folate, iron and thiamin [27,28].

STORAGE: Refrigerate in an airtight container.

USAGE: Can be used in a flour blend or by itself.

Millet: An ancient cereal grain that gives a light beige or yellow color to foods. It is easy to digest and has a mildly sweet, nut-like flavor. Millet creates lighter baked goods and is high in fiber, protein, and nutrients. It is a great option for baked goods that need structure, but also require yeast. Millet has a short shelf life and can become rancid/bitter [29].

STORAGE: Refrigerate or freeze in an airtight container.

USAGE: No more than 25 percent of any flour blend.

Oat: Oat flour can be made by grinding oats in a food processor if unable to find it in a store. Oats are high in fiber, protein and nutrition. Oats and oat flour can add structure, taste,and texture to a variety of baked goods. One major concern regarding oats and gluten intolerances is in North America oats and wheat can be rotated in fields, making cross-contamination a great concern [30]. Make sure to use 
oats that are marked "gluten-free" to help avoid this.

STORAGE: Cool, dry place or freezer in an airtight container.

USAGE: Less than 30 percent of a flour blend.

Rice: There are three main varieties of rice flour, brown (higher in fiber), sweet rice (higher in starch), and white. The texture can be coarse, medium, or fine. Fine is used mainly for more delicate baked goods. Medium grind is more readily available, and suitable for most baking. Coarse is best for cereals and coatings. Rice is easy to digest and blend, with white rice having a very bland taste, and brown having a slightly nutty flavor. If too much is used within a baked good, the item can become crumbly.

STORAGE: Cool, dry place in airtight container. Brown rice flour should be refrigerated in an airtight container.

USAGE: Best combined with other flours [31].

Sorghum: Many believe sorghum flour is similar in taste to wheat and is available in red and white varieties. Can impart a slightly sweet taste and gives a whole-wheat appearance to baked goods. Sorghum is high in protein, fiber, phosphorus, potassium, and Vitamin B. It provides structure and can also be called milo or jowar.

STORAGE: Room temperature or refrigerate in an airtight container

USAGE: Should be no more than 25-30 percent of flour blend Grasses

Buckwheat: Commonly mistaken for wheat, it is actually a fruit from the same family as rhubarb and sorrel. Buckwheat has a strongflavor but is preferred for baking. It comes in light, medium and dark varieties and is high in fiber, amino acids, protein and $B$ vitamins [32].

STORAGE: Refrigerate in an airtight container

USAGE: For bread/rolls, use up to $1 / 2$ cup buckwheat flour to $1 / 2$ to 1 cup all-purpose flour.

Wild Rice: Often mistaken for rice, wild rice is actually an aquatic grass originally found in lakes (particularly in Minnesota). Wild rice is rich in folate and has a long shelf life due to being dried and slightly fermented. It has a very dark brown/black color that can add a rich hue to baked goods. Wild rice has an interesting flavor and texture and is best used as part of a flour blend. It can also be used to thicken casseroles, stews, gravies, etc [32].

STORAGE: Cool, dry place in an airtight container

USAGE: Add up to 25 percent to a flour blend

Almond: Can be made by finely grinding blanched almonds in coffee grinder/food processor, but can quickly turn into almond butter, so take care not to over-grind. Almonds have a sweet, nutty flavor, add texture and structure to baked goods. It is high in protein, fiber, vitamin $\mathrm{E}$ and provides a healthy source of fats. Because of its high-fat content, almond flour can go rancid quickly. Almond flour can also add moisture to finished products.
STORAGE: Refrigerate or freeze in an airtight container

USAGE: Add up to 25 percent in basic flour blend. Up to 50 percent or more in cakes leavened with eggs.

Coconut: A low-carb, high-fiber flour that is tolerated well by people with multiple allergies. Coconut flour has a sweet flavor and is very dense. It absorbs extra liquid so when using, be aware that your recipe may need adjusting or may call for extra liquids. When using 100 percent coconut flour, adding extra eggs can help prevent a dense end product [32,33].

STORAGE: Room temperature or refrigerate in an airtight container

USAGE: For best results, add up to 15 percent in a flour blend, but able to use 100 percent

\section{Seeds}

Flaxseed: Whole flaxseeds are indigestible, so must be ground. They are high in fiber and omega- 3 fatty acids and give a flecked or speckled appearance to baked items. Too much flax can cause a laxative effect in some people, so add to recipes sparingly.

STORAGE: Refrigerate or freeze in an airtight container

USAGE: Add 2-3 tbsp per recipe for baked goods; can sprinkle on yogurt or cereal for a nutritional boost

Hemp: Whole grain flour that is high in protein and dietary fiber. It contains all the essential amino acids and imparts a nutty flavor. Too much of a mixture can give a gritty texture and an unpleasant taste.

STORAGE: Refrigerate or freeze in an airtight container

USAGE: Add $1 / 4$ to $1 / 2 C$ to basic flour blend

Quinoa: An easy to digest flour that has a delicate, nutty flavor, similar to wild rice. Quinoa contains high levels of calcium, protein, phosphorus, iron, fiber, B vitamins and complex carbohydrates.

STORAGE: Refrigerate or freeze in an airtight container

USAGE: Up to 25 percent of total flour blends

\section{Tubers/Roots}

Potato flour: This flour is not to be mistaken for potato starch. Potato flour is made from dehydrated potatoes and is a fine yellow-white powder. It is high in fiber and protein. It gives a soft, chewy mouth-feel to baked goods and pizza crusts. It can be used in place of xanthan gum or guar gum. Too much in a recipe can create a gummy final product [34].

STORAGE: Cool, dry place in an airtight container

USAGE: Add 2-4 tbsp per recipe, reduce/eliminate the gum ingredients accordingly

Sweet potato: Sweet potato flour, with its yellow/orange color, tends to color baked items and has a taste that works well with chocolate, spices, molasses, etc. Using too much sweet potato flour can cause a gummy texture, but in smaller amounts gives a great chewy texture and helps with the browning of baked goods. 
STORAGE: Room temperature, in an airtight container

USAGE: Up to 25 percent of a flour blend

Cassava: While cassava and tapioca come from the same plant, they are NOT the same. Tapioca is extracted from the starch of the cassava plant, while cassava flour is the whole root, which is dried, peeled and ground. Cassava flour has a very neutral flavor and is not grainy or gritty in texture. Cassava flour doesn't need to be blended with other flours, as it can replace wheat flour 1:1 in the majority of recipes. It is high in carbohydrates and is good for pasta, bread, and tortillas [25].

\section{Starches}

In gluten-free baking, starches are vital to having a successful final product. They help with rebuilding the binding, texture, and structure that is lacking. They can also be used to thicken gravies, sauces or puddings. There are two main classes of starches and they behave differently depending on the item being made.

Grain-based (Corn): A flavorless powder that lightens baked goods and creates an airy texture. It has very little nutritional value and can be used as a thickener for gravies or sauces. It gelatinizes at a very high temperature as compared to tuber/ root starches. Cornstarch can cause sauces to look opaque or matte and can cause cereal like a flavor once cooked. It is best used for longer thickening cooks, like stews.

Tuber/Root-based: Often larger grained than grain-based starches and gelatinize at a lower temperature. Sauces thickened with these starches are more translucent and glossy and have a silkier mouthfeel. These starches also impart less of a flavor once cooked. They are best used toward the end of cooking, or in custards, puddings or pie fillings.

Potato: This is different than potato flour. It is made from dehydrated potatoes, but only the starch portion. It is often used as a 1:1 cornstarch substitution in baking. It works great in baking, especially when combined with eggs. It must be stirred before measuring as it has a tendency to clump up. Often used in flour blends at $1 / 2$ to $3 / 4$ Cup.

Tapioca: Extracted from the cassava root through a process of washing and pulping, this is what remains after the pulp is squeezed and the liquid dries. Helps with creating a crispy crust or a chewy texture in baking.

Arrowroot: A great alternative to cornstarch, it can be used to thicken sauces or gravies and imparts no taste, while leaving the sauces glossy and clear. It can be used as a coating to create a crispy outer layer (ex: French fries/fried chicken). It breaks down at higher temperatures, so it is best added toward the end of cooking $[35,36]$.

\section{Binding agents}

Majority of gluten-free recipes call for a binding agent. These ingredients are used to bind, thicken, or emulsify gluten-free ingredients [37].

Guar gum: A highly soluble fiber that comes from guar beans.
Guar gum is a great emulsifier, but not great at gelling. Sometimes it can impart a bean-like flavor onto finished product but really depends on other ingredients [38,39].

Xanthan gum: Made by fermenting corn with a microbial microbecalled "Xanthomonas campestris." Due to this being a man-made product, the taste is controlled, oftentimes this has no flavor impact on finished products. When adding this to recipes, it is important not to overmix after it is added, as too much mixing may cause a rubbery or tough final texture [39].

Psyllium husk: A fiber that is created by grinding the outer husks of the psyllium plant. It provides a great amount of soluble fiber and is very good at retaining moisture in recipes. It swells as it's exposed to fluids so it can change the texture of cooked foods [39].

These binders are optional, and many choose to eliminate them due to potential gastrointestinal that can be experienced. But if they are used, here are a few guidelines:

For cakes, cookies, bars, muffins and quick bread: $1 / 2$ tsp per cup of flour

Yeast bread or any items that call for yeast: 1 tsp per cup of flour

Pizza dough or pie crust: $1 \frac{1}{2}$ tsp per cup of flour

\section{Creating a flour mixture}

Gluten-free baking requires a mixture of at least two flours. Typically mixtures are comprised of at least one starch and one flour. Starches help to create the binding, texture and structure usually provided by gluten. Flours are separated into three main categories based on their nutrition content, density, and ability to blend with other flours.

Light: Typically comprised of the starches (arrowroot starch, corn starch, potato starch, tapioca starch)

Medium: Flours that are lighter when used and are more stable when paired with just a starch (bean flours, millet, oat, quinoa, sorghum, cassava, and white rice)

Heavy: Denser, more nutritious flours that are rarely used alone, and must be used with another medium-based flour (almond, amaranth, brown rice, buckwheat, coconut, corn, wild rice, sweet potato, potato, pea, hemp, and flax)

There are four main formulas that can be used when looking to create a gluten-free flour blend:

Combo \# 1: 1 Medium flour + 1 or 2 starches

Combo \#2: 2 Medium flours + 1 or 2 starches

Combo \#3: 1 Medium flour +1 Heavy flour +1 or 2 starches

Combo \#4: A variety of Medium and Heavy flours +1 or 2 starches.

\section{Miscellaneous}

Here are a variety of other items that may potentially be substituted based on personal preference or other dietary restrictions not as common. 
Oil substitutions: Oils are typically a 1:1 swap in recipes, just be wary because will impart flavors and thus potentially change the taste profile of the finished product.

Sugar substitutions: There are a variety of different sugars that can be used in place of white cane sugar. This is just a partial list of information on the most commonly used sugar substitutes [40].

Artificial sweeteners: Acesulfame potassium (Sunett/Sweet One), Aspartame (Equal, NutraSweet), Neotame, Saccharin (SugarTwin/Sweet'N Low) [41].

Sucralose (Splenda): For super high sugar recipes that rely on sugar for texture, you should only replace about 25 percent of sugar with sucralose. When making yeast products, the recipe may need a small amount of regular sugar to activate the yeast. In most recipes, sucralose is a 1:1 trade with white sugar.

Sugar slcohols: Hydrogenated starch hydrolysate, Isomalt, Lactitol, Maltitol, Mannitol, Sorbitol

Erythritol: Typically contains 75 percent the sweetening power of sugar, and is best used in items that will be consumed within 24 hours, as the sweetening effect will fade. Erythritol can cause crystallization and dryness, so it's best to use no more than $1 / 2$ cup in a recipe.

Xylitol: Xylitol has fewer calories than white sugar and does not raise blood sugar or insulin levels, it also contains no fructose. It can improve calcium absorption but eat too much of it can cause digestive side effects. It is also highly toxic to dogs.

\section{Novel sweeteners: Tagatose (Naturlose), Trehalose}

Stevia: Stevia works great under high temperatures, and combines well with fruits. However, it has a different texture than sugar. Stevia won't work in recipes requiring yeast. To replace white sugar use, $1 \mathrm{tsp}$ of Stevia should be used to every 1 cup white sugar.

Natural sweeteners: Agave nectar, date sugar, fruit juice concentrate, molasses, maple sugar

Honey: Honey is best used in cold/raw desserts because once its cooked it loses its antioxidant, enzymes and nutrients naturally contained. Honey can cause baked goods to brown faster, so it may be prudent to reduce cooking temperature by 10-25 degrees. To replace white sugar use $3 / 4$ Cup to every 1 Cup white sugar, while also reducing wet ingredients by $1 / 4$ Cup.

Monk sruit: Pure extract is 300 times sweeter than sugar, but doesn't bake well. Works best with cold or raw items where you don't need sugar to give texture. To replace white sugar use $1 / 4-1$ Cup to every 1 Cup white sugar (start with less and gradually add)

Maple Syrup: It is important to read the label and only use $100 \%$ pure maple syrup as a sugar replacer. It also can impart a distinct taste onto finished goods so make sure to pair with flavors that will work well. To replace white sugar use $2 / 3$ Cup to every 1 Cup white sugar, while reducing the liquids by about $25 \%$.
Coconut sugar: Coconut sugar is very similar to brown sugar in taste and also contains a variety of antioxidants, vitamins and minerals. It can also help with blood sugar levels as it has a low glycemic index, but is very high in fructose and calories.

Corn products: Corn is included in a variety of forms in many different commercial products. People with allergies/ intolerances to corn may require some of these substitutions.

Baking powder: Simply use the following for every $1 \mathrm{tsp}$ of baking powder use 1 tsp of baking soda $+1 / 2$ tsp cream of tartar.

Powdered sugar: Many powdered sugars contain cornstarch. There are a few brands that don't so be sure to always read labels before assuming it is/isn't included. To make powdered sugar simply grind 1 Cup granulated sugar +1 tbsp corn-free starch. NOTE: This won't be quite as fine as commercial brands

Vanilla extract: You can buy alcohol/corn free vanilla extract or make your own by adding 2 vanilla beans into a small bottle of potato vodka and letting steep for 1-2 months, then remove the beans and use.

Corn syrup: Majority of liquid sweeteners (honey, agave, cane syrup) will sub for corn syrup or simply make your own by combining 1 Cup granulated sugar and $1 / 4$ Cup water for every 1 cup corn syrup.

\section{Conclusion}

There are many substitutions available for common allergen causing food ingredients. The home food production operations could utilize information given in this guide to prepare food for consumers with food allergies and sensitivities.

\section{References}

1. Boyce J, Assaad A, Burks A, et al. Guidelines for the Diagnosis and Management of Food Allergy in the United States: Report of the NIAIDSponsored Expert Panel. J Allergy Clin Immunol. 2010; 126(6): S1-S58. doi: 10.1016/j.jaci.2010.10.007

2. Pongdee T. Food Allergy versus Food Intolerance. AAAAI. 2011.

3. Jackson KD, Howie LD, Akinbami ப. Trends in Allergic Conditions Among Children: the United States, 1997-2011. NCHS Data Brief. 2013; 1-8.

4. Kamdar TA, Peterson S, Lau CH, Saltoun CA, Gupta RS, Bryce PJ. Prevalence and Characteristics of Adult-Onset Food Allergy. J Allergy Clin Immunol Pract. 2015; 3(1): 114-115.e1. doi: 10.1016/j.jaip.2014.07.007

5. Keet CA, Savage JH, Seopaul S, Peng RD, Wood RA, Matsui EC. Temporal Trends and Racial/Ethnic Disparity in Self-Reported Pediatric Food Allergy in the United States annals of Allergy. Ann Allergy Asthma Immunol. 2014; 112(3): 222-229.e3. doi: 10.1016/j.anai.2013.12.007

6. Gupta R, Springston E, Warrier M, et al. The Prevalence, Severity, and Distribution of Childhood Food Allergy in the United States. Pediatrics. 2011; 128(1): e9-e17. doi: 10.1542/peds.2011-0204

7. Food Allergy Research \& Education. Facts and Statistics. 2018.

8. Bock S, Munoz A, Hugh A, Sampson M. Fatalities Due to Anaphylactic Reactions to Foods. J Allergy Clin Immunol. 2001; 107(1): 191-193. doi: 10.1067/mai.2001.112031

9. Bock S, Munoz A, Hugh A, Sampson M. Further Fatalities Caused by Anaphylactic Reactions to Food. J Allergy Clin Immunol. 2006; 119(4): 1016-1018. doi: 10.1016/j.jaci.2006.12.622 
10. Herbert L, Shermesh E, Bender B. Clinical Management of Psychosocial Concerns Related to Food Allergy. J Allergy Clin Immunol Pract. 2016; 4(2): 205-213. doi: 10.1016/j.jaip.2015.10.016

11. Bollinger M, Dashlquist L, Mudd K, Sonntag C, Dillinger L, McKenna K. The Impact of Food Allergy on the Daily Activities of Children and Their Families. Ann Allergy Asthma Immunol. 2006; 96(3): 415-421. doi: 10.1016/ S1081-1206(10)60908-8

12. Sampson $H$, Mendelson $L$, Rosen J. Fatal and Near-Fatal Anaphylactic Reactions to Food in Children and Adolescents. N Engl J Med. 1992; 327(6): 380-384. doi: 10.1056/NEJM199208063270603

13. Clark S, Espinola J, Rudders S, Banerji A, Camargo C. Frequency of US Emergency Department Visits for Food-Related Acute Allergic Reactions. J Allergy Clin Immunol. 2011; 127(3): 682-683. doi: 10.1016/j. jaci.2010.10.040

14. Cara C. Your Guide to Allergen-Friendly Baking: Egg Substitutes. Fork and Beans. 2018.

15. Breanna T. The 'Gelatin Egg' Egg Substitute. He Won't Know It's Paleo. 2018.

16. Cara C. Guide to Understanding Dairy Substitutes. Fork and Beans. 2013.

17. Kids with Food Allergies. Milk Allergy? How to Substitute Milk in Recipes. 2018.

18. Nicole L. Homemade Sweetened Condensed Milk - Cheap, Easy, Even Dairy Free! 2018.

19. The Olive Crush. Cooking With Olive Oil Guide. 2018.

20. Christensen E. What's the Difference? Flour, Cornstarch, Potato Starch, and Arrowroot. Kitchn. 2018.

21. Chistmas N. Which Gluten-Free Flour Should You Use? All recipes. 2018.

22. Raffensperger S. How to Use 23 Different Gluten-Free Flours. FaveGlutenFreeRecipes.Com. 2016

23. Gay B. How to Use Sugar Substitutes for Baking. The good stuff. 2015.

24. Cara C. Guide to Gluten-Free Flours. Fork and Beans. 2018.
25. Bryan L. 5 Things You Need to Know About Cassava Flour. Downshiftology. 2017.

26. Hand J. How to Bake with Sugar Substitutes. Bulletproof. 2018.

27. Mace M. Binders in Gluten-Free Baking. MiMFiT. 2018.

28. Chelsea S. Gluten Free Flours: Overview of the Most Popular GF Flours. 2018.

29. Morley B. Gluten Free Flour Substitutions. Fave Gluten Free Recipes.Com 2018.

30. The American Academy of Allergy, Asthma \& Immunology. Food Intolerance AAAAI. 2018.

31. The club, trim down. How to Use 24 Different Gluten-Free Flours in the Kitchen? Trim Down Club. 2015

32. Allergy Free Cooking, Baby! Allergy Free Cooking Substitutes. 2018.

33. Dermer D. Allergen-Friendly Baking Substitutions. Gluten Free \& More. 2018.

34. Lane C. Guar Gum or Xanthan Gum: Which One Should You Use? Gluten Free Bread. 2018.

35. Bryan L. 5 Things You Need to Know About Arrowroot Powder. Downshiftology. 2017.

36. Kids with Food Allergies. How to Avoid Corn and Make Corn-Free Recipes. 2018.

37. Hilson B. 25 Gluten-Free Baking Solutions Gluten Free \& More. Gluten Free and More. 2018.

38. Gruss T. How to Use Xanthan Gum and Guar Gum in Gluten-Free Cooking The Spruce. 2018.

39. St Claire T. What's The Difference between Xanthan Gum \& Guar Gum? GlutenFreeClub. 2012.

40. McDonell K. 8 Natural Substitutes for Sugar. Healthline. 2017.

41. Mayo Clinic. Pros and Cons of Artificial Sweeteners. 2018. 